\title{
Use of thiazide diuretics for the prevention of recurrent kidney calculi: a systematic review and meta-analysis
}

Dan-feng Li ${ }^{1 \dagger}$, Yu-lu Gao ${ }^{2 \dagger}$, Hong-chao Liu ${ }^{1}$, Xiao-chen Huang ${ }^{1}$, Rui-fang Zhu ${ }^{3 *}$ and Chang-tai Zhu ${ }^{1 *}$

\begin{abstract}
Background: Thiazide diuretics reduce the risk of recurrent kidney calculi in patients with kidney calculi or hypercalciuria. However, whether thiazide diuretics can definitely prevent recurrent kidney calculi remains unclear. We aimed to evaluate the effect and safety of thiazide diuretics on recurrent kidney calculi.

Methods: The PubMed, Cochrane Library, and EMBASE databases were systematically searched using the keywords thiazide diuretics and kidney calculi to identify randomized controlled trials (RCTs). The primary outcome was the incidence of recurrent kidney calculi, and the secondary outcome was the 24-h urinary calcium level. The pooled risk ratio $(\mathrm{RR})$, risk difference $(\mathrm{RD})$, standardized mean difference $(\mathrm{SMD})$, and 95\% confidence interval (Cl) were calculated. The evidence quality was graded using the GRADE criteria, and recommendations for recurrent kidney calculus prevention using thiazide diuretics were reassessed.
\end{abstract}

Results: Eight RCTs involving 571 patients were included. The pooled RR for the incidence of kidney calculi in the thiazide diuretic groups was $0.44(95 \% \mathrm{Cl} 0.33-0.58, P<0.0001)$ compared to that in the placebo and untreated groups; the pooled RD was $-0.23(95 \% \mathrm{Cl}-0.30$ to $-0.16, P<0.0001)$. The pooled SMD for the 24 -h urinary calcium level was $-18.59(95 \% \mathrm{Cl}-25.11$ to $-12.08, P<0.0001)$. The thiazide diuretic groups had a high incidence of adverse reactions and low tolerance. The evidence quality for decrease in kidney calculus incidence using thiazide diuretics was low, while that for the 24-h urinary calcium level decrease among those with recurrent kidney calculi was moderate, and that for the decrease in kidney calculus incidence using short-acting and long-acting thiazide diuretics was low. The overall strength of recommendation for prevention of recurrent renal calculi using thiazide diuretics was not recommended. The subgroup and sensitivity analysis findings were robust.

Conclusions: Long-term use of thiazide diuretics reduces the incidence of recurrent renal calculi and 24-h urinary calcium level. However, the benefits are insufficient, and the evidence quality is low. Considering the adverse effects, poor patient compliance, and economic burden of long-term medication, their use in preventing recurrent kidney calculi is not recommended.

Keywords: Thiazide diuretics, Kidney calculi, Randomized controlled trial, Placebo

*Correspondence: zct101@163.com; ruifang.zhu@sxmu.edu.cn

${ }^{\dagger}$ Dan-feng Li and Yu-lu Gao contributed equally to this work

${ }^{1}$ Department of Transfusion Medicine, Shanghai Jiao Tong University Affiliated Sixth People's Hospital, Shanghai 200233, China

${ }^{3}$ School of Nursing, Shanxi Medical University, 56 Xinjian South Road, Yingze District, Taiyuan 030001, Shanxi, China

Full list of author information is available at the end of the article

\section{Background}

Kidney calculi are a common urinary system disease, and their incidence is increasing annually [1]. In the clinical guideline of the American College of Physicians, the prevalence of kidney calculi in men and women was $13 \%$ and $7 \%$, respectively, and the 5 -year recurrence rate of untreated kidney calculi was $35-50 \%$ [2].

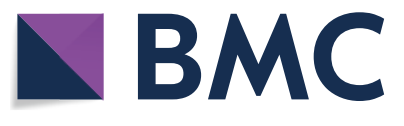

(c) The Author(s) 2020. This article is licensed under a Creative Commons Attribution 4.0 International License, which permits use, sharing, adaptation, distribution and reproduction in any medium or format, as long as you give appropriate credit to the original author(s) and the source, provide a link to the Creative Commons licence, and indicate if changes were made. The images or other third party material in this article are included in the article's Creative Commons licence, unless indicated otherwise in a credit line to the material. If material is not included in the article's Creative Commons licence and your intended use is not permitted by statutory regulation or exceeds the permitted use, you will need to obtain permission directly from the copyright holder. To view a copy of this licence, visit http://creativeco mmons.org/licenses/by/4.0/. The Creative Commons Public Domain Dedication waiver (http://creativecommons.org/publicdomain/ zero/1.0/) applies to the data made available in this article, unless otherwise stated in a credit line to the data. 
Correspondingly, the high recurrence rate yielded a certain economic burden to patients with kidney calculi. Kidney calculi are caused by abnormal accumulation of some crystalline substances, such as calcium, oxalic acid, uric acid, and cystine, and organic matrices, such as matrix A and acid mucopolysaccharide, in the kidney. Most kidney calculi are composed of calcium; calcium oxalate stones are the most common, accounting for $74.8 \%$ of all cases of stones [3]. In response to increasing incidence of kidney calculi, various interventions have been performed to prevent the occurrence of stones, including dietary interventions and medical treatments [4-6]. Among them, thiazide diuretics are the commonly used drugs for preventing recurrent kidney calculi $[7,8]$. However, some clinical trials reported that thiazide diuretics had no significant prophylactic effect compared with the placebo $[9,10]$. We also found that the recommended grade of thiazide diuretics to prevent recurrent kidney calculi was not consistent among guidelines [2, $7,8,11]$. Further, no meta-analyses have yet been conducted to prove that thiazide diuretics can prevent recurrent renal calculi. Therefore, we performed a systematic review and meta-analysis of clinical trials that investigated thiazide diuretics for the prevention of recurrent renal calculi to provide evidence-based medical data for use in clinical practice. We aimed to evaluate the effect and safety of thiazide diuretics on recurrent kidney calculi patients, comparing the incidence of recurrent kidney calculi and the 24-h urinary calcium level with that of placebo or no medication group in randomized controlled trials (RCTs).

\section{Methods}

Trial search

Two researchers independently searched all studies with keywords of thiazide diuretics and kidney calculi published in the PubMed, EMBASE, and Cochrane Library databases. The following search terms were used: "kidney stones", "kidney calculi", "renal calculi", "nephrolith", "thiazide diuretics", "sodium chloride cotransporter inhibitors". The article search was limited by study design of RCTs. The search was performed until December 31, 2018. In addition, the references of the included studies were also retrieved to supplement the relevant research, including gray literatures (e.g., clinical trials). When the opinions of the two researchers differed, a third researcher was consulted. When there were other languages used, we sought help from linguists.

\section{Inclusion and exclusion criteria}

The inclusion and exclusion criteria for the studies were determined prior to data extraction. The inclusion criteria included the following: (1) RCTs with thiazide diuretic administration as the intervention and placebo or no medication as the control condition; (2) patients with renal calculi or hypercalciuria as the study subjects; and (3) test indicators including at least one of the following: number of patients with new stones, 24-h urinary calcium level, 24-h urinary oxalate level, and serum calcium level. Conversely, the exclusion criteria included the following: (1) non-RCTs; (2) RCTs without a treatment group or placebo group; (3) trials with incomplete or no data available; and (4) other study types, e.g., abstract, case report, and review.

\section{Risk of bias assessment}

Two investigators independently searched for studies using different search strategies and screened for studies that met the inclusion criteria. The researchers evaluated the quality of the included RCTs using the Cochrane Library risk bias assessment tool. The seven items used to assess bias in each trial included random sequence generation, allocation concealment, double blindness of participants and trial performers, blindness of outcome assessment, incomplete outcome data, selective reporting, and other biases. Each quality item was divided and categorized into high risk, undefined risk, and low risk. The quality of the included trials was rated as low quality, high quality, or medium quality according to the following criteria: (1) if random sequence generation or allocation concealment was assessed to be of a high risk, the trial would be considered to be of low quality regardless of the risk of other projects; (2) if random sequence generation and allocation concealment were assessed to be of a low risk, and all other items were assessed to be of a low or an undefined risk, the test would be of high quality; and (3) if the tests did not meet the high risk or low risk criteria, the quality of the trial was considered moderate [12].

\section{Data extraction}

Two investigators extracted the following data from the included studies: primary author; year of publication; sex, age; sample size; interventions; control group; number of lost visits; and follow-up time. When the trials were greater than two sets or had multi-factor designs, we only extracted content relevant to this study.

\section{Statistical analysis}

We performed a meta-analysis and calculated the relative ratio (RR), risk difference (RD), standardized mean difference (SMD), and 95\% confidence interval (CI). The pooled RR and RD were used to estimate the efficiency of thiazide diuretics on recurrent kidney calculi by using Mantel-Haenszel method. The pooled SMD were used to evaluate the effect of thiazide diuretics 
on 24-h urinary calcium level by using inverse variance method. We used $I^{2}$ statistics to assess statistical heterogeneity. An $I^{2}$ value of $0-25 \%$ indicates no significant heterogeneity; $26-50 \%$, low heterogeneity; $51-75 \%$, moderate heterogeneity; and $>75 \%$, high heterogeneity [13]. Data were pooled using the fixed-effects model when the $I^{2}$ value was $<50 \%$; data were pooled using the random-effects model when the $I^{2}$ value was $>50 \%$. The test level was set at $\alpha=0.05$; $P$ values of 0.05 were considered to indicate that the difference was statistically significant. We used the Review Manager 5.2 software (The Cochrane Collaboration, Oxford, UK) to perform the meta-analysis and forest plot analysis and the Stata 13.0 software (Stata Corp, College Station, TX) to conduct the publication bias test (Egger's test). Evidence quality grading was performed for each outcome measure with reference to the GRADE criteria, and recommendations for the prevention of recurrent kidney calculi using thiazide diuretics were reassessed on the basis of a decision table formed according to the recommendations of the WHO Handbook for Guideline Development [14]. To assess whether the efficacy of thiazide diuretics in preventing recurrent kidney calculi is related to their clinical features, we performed a subgroup analysis based on the duration of drug action; we also did a subgroup analysis based on quantitative methods of 24-h urinary calcium. For the robustness of the results, we conducted a sensitivity analysis.

\section{Results}

\section{Study search}

A total of 103 records were searched according to the search strategy (Additional file 1: Table S1), and 28 of them were related to thiazide diuretics for preventing kidney calculi after screening of the titles and abstracts (Fig. 1). Eight of them were reviews; seven investigated non-thiazide diuretics compared with a control condition; two reported failure to reduce the incidence of renal calculi; two were meta-analyses; three were non-RCTs; and six reported reduction of the occurrence of kidney calculi. We finally included eight RCTs conducted on thiazide diuretics $[9,10,15-20]$.

\section{Characteristics and risk of bias}

Among the included articles, there were seven studies published in English language [9, 10, 15-17, 19, 20] and one in Spanish language [18]. Seven of them had patients with recurrent calcium stones as the subjects $[9,10,16-$ 20]; one trial had patients with idiopathic hypercalciuria as the subjects [15]. Table 1 shows the specific characteristics and data of the studies included. The patients and experimenters were both blinded to the study data. Seven papers did not report the generation of random sequence $[9,10,15-18,20]$ and were of moderate quality. One study used the medical record number to assign patients into groups [19], which was of low quality. The overall quality of the studies was moderate (Fig. 2).

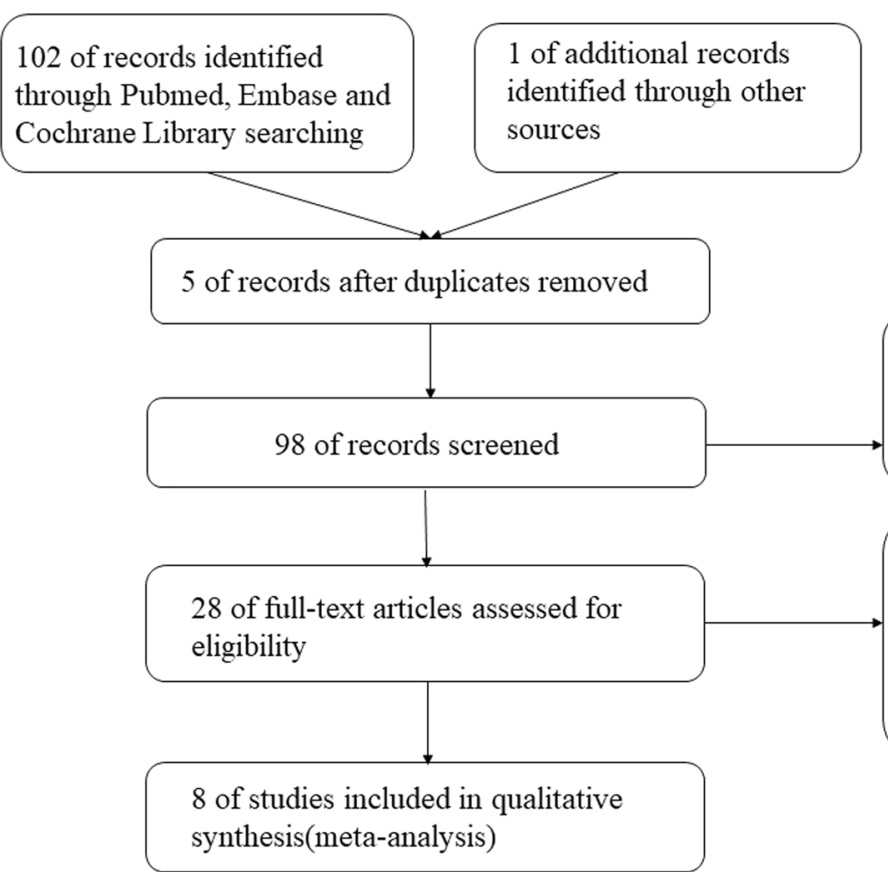

70 of records were excluded based on titles and abstracts for not fulfilling inclusion criteria on basis of type of article and study design

20 of full-text articles were excluded because they were not RCTs that were complied with included criteria(eight of them were reviews; seven investigated non-thiazide diuretics; two were metaanalyses; three were non-RCTs)

8 of studies included in qualitative (meta-analysis)

Fig. 1 Literature search and screening process 


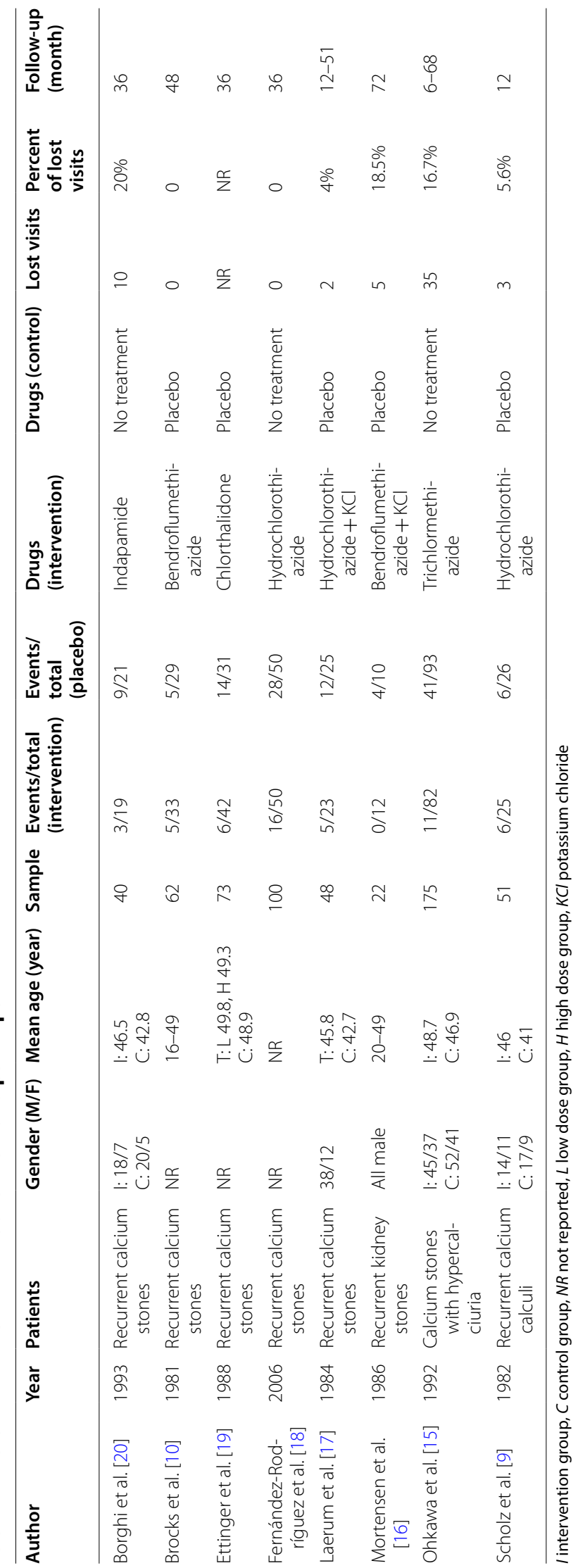




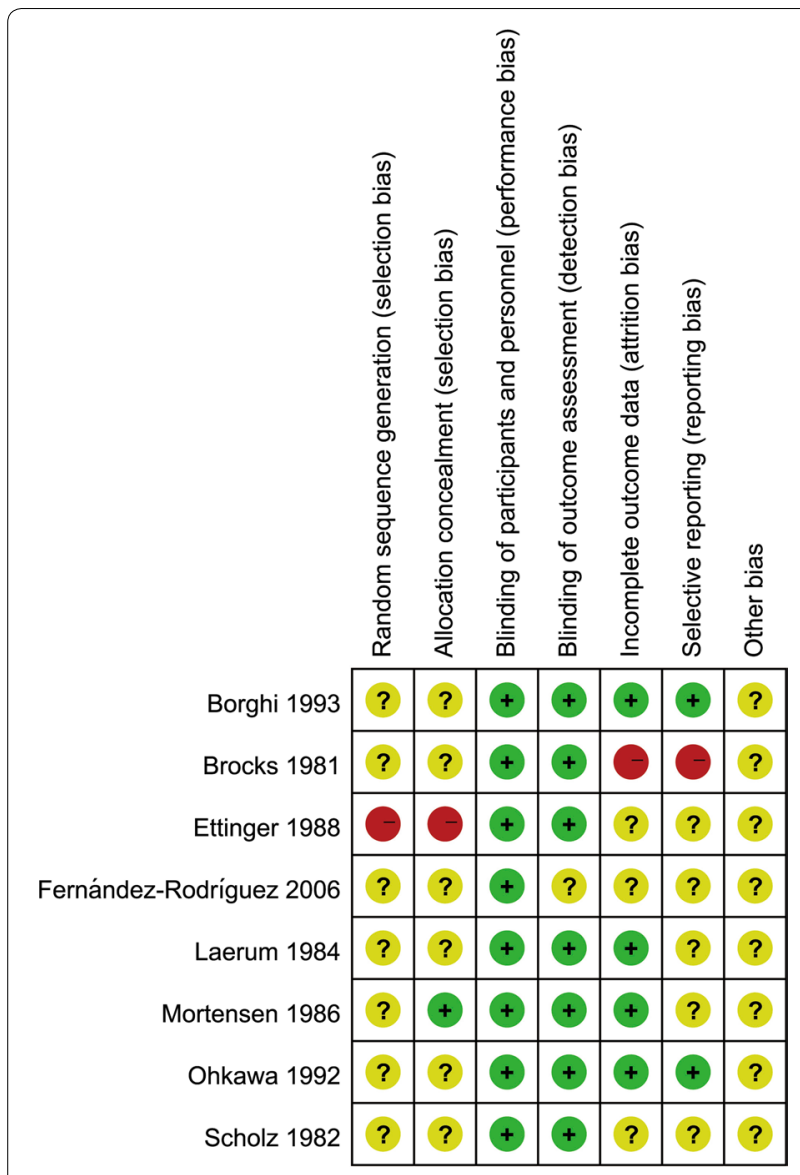

Fig. 2 Risk of bias summary for included studies

\section{Incidence of recurrent stone events}

There were 286 cases of patients with recurrent kidney calculi in the thiazide diuretic groups and 52 cases of new stones, accounting for $18.2 \%$ of all patients; conversely, there were 285 cases in the placebo and untreated groups and 119 cases of new stones, accounting for $41.2 \%$ of all patients. The pooled RR for the incidence of renal calculi in the thiazide diuretic groups was 0.44 (95\% CI $0.33-$ 0.58, $P<0.0001, I^{2}=21 \%$; fixed-effects model; Fig. 3); the pooled RD was $-0.23(95 \% \mathrm{CI}-0.30$ to -0.16 , $P<0.0001, I^{2}=43 \%$; fixed-effects model; Fig. 4).

\section{Twenty-four-hour urinary calcium level}

The pooled SMD for the 24-h urinary calcium level was -18.59 (95\% CI -25.11 to $-12.08, P<0.0001, I^{2}=99 \%$; random-effects model; Fig. 5). Furthermore, Mortensen et al. [16] reported that the 24-h urinary calcium level of seven patients in their thiazide diuretic group was reduced by $20-25 \%$; none of their control group patients had a urinary calcium level reduction of $>20 \%$.

\section{Subgroup analyses}

The subgroup analysis showed that there was no significant difference in the incidence of recurrent renal calculi between short-acting $[9,17,18]$ and long-acting thiazide diuretics $[10,15,16,19,20](P=0.05$; Fig. 6). Subgroup analysis revealed that there was a significant difference between 24-h urinary calcium tested by using calcium mass $(\mathrm{mg})$ and the ratio with creatinine $(\mathrm{mol} / \mathrm{mol})$ $(P<0.00001$, Fig. 7$)$.

\section{Sensitivity analysis}

The sensitivity analysis showed that the results did not change significantly after changing the fixed-effects model to the random-effects model (Figs. 8 and 9).

\section{Adverse reactions}

Borghi et al. [20] reported that two patients in their thiazide diuretic group did not complete the study because of the development of hypotension and hypokalemia; Ettinger et al. [19] reported that $41.5 \%$ of the patients in

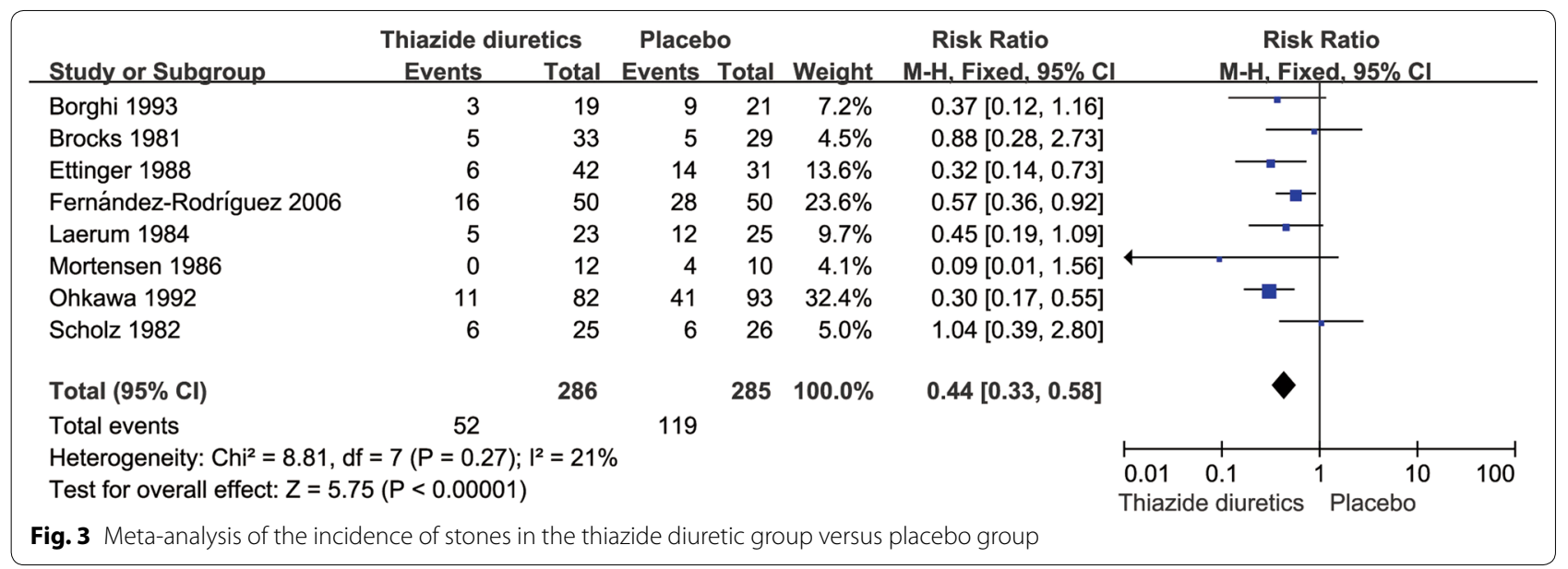




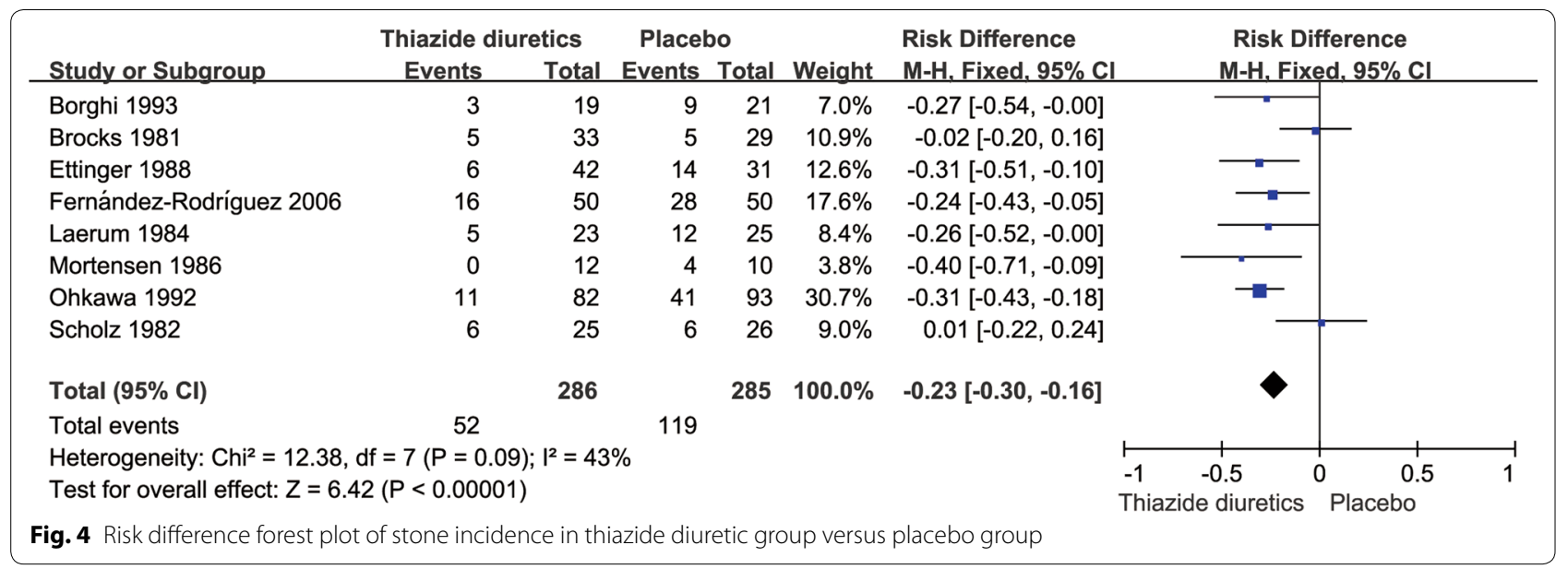

\begin{tabular}{|c|c|c|c|c|c|c|c|c|c|}
\hline \multirow[b]{2}{*}{ Study or Subgroup } & \multicolumn{3}{|c|}{ Thiazide diuretics } & \multicolumn{2}{|r|}{ Placebo } & \multirow[b]{2}{*}{ Total } & \multirow[b]{2}{*}{ Weight } & \multirow{2}{*}{$\begin{array}{l}\text { Std. Mean Difference } \\
\text { IV. Random, } 95 \% \mathrm{CI}\end{array}$} & \multirow{2}{*}{$\begin{array}{l}\text { Std. Mean Difference } \\
\text { IV. Random, } 95 \% \mathrm{CI}\end{array}$} \\
\hline & Mean & SD & Total & Mean & SD & & & & \\
\hline Borghi 1993 & -217 & 147.037 & 19 & -45 & 132.449 & 21 & $27.7 \%$ & $-1.21[-1.89,-0.53]$ & 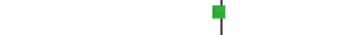 \\
\hline Ettinger 1988 & -75 & 26 & 19 & 0.005 & 0.001 & 31 & $27.6 \%$ & $-4.64[-5.74,-3.53]$ & - \\
\hline Ohkawa 1992 & -0.155 & 0.001 & 82 & -0.062 & 0.001 & 93 & $17.0 \%$ & $-92.60[-102.41,-82.78]$ & 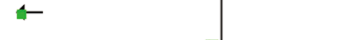 \\
\hline Scholz 1982 & -53 & 9.669 & 25 & -1 & 13.65 & 26 & $27.6 \%$ & $-4.31[-5.34,-3.28]$ & 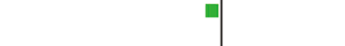 \\
\hline \multirow{2}{*}{\multicolumn{8}{|c|}{ 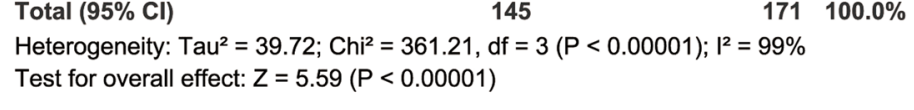 }} & $-18.59[-25.11,-12.08]$ & \\
\hline & & & & & & & & & $\begin{array}{ccccc}-50 & -25 & 0 & 25 & 50 \\
\text { Thiazide diuretic } & \text { Placebo }\end{array}$ \\
\hline
\end{tabular}

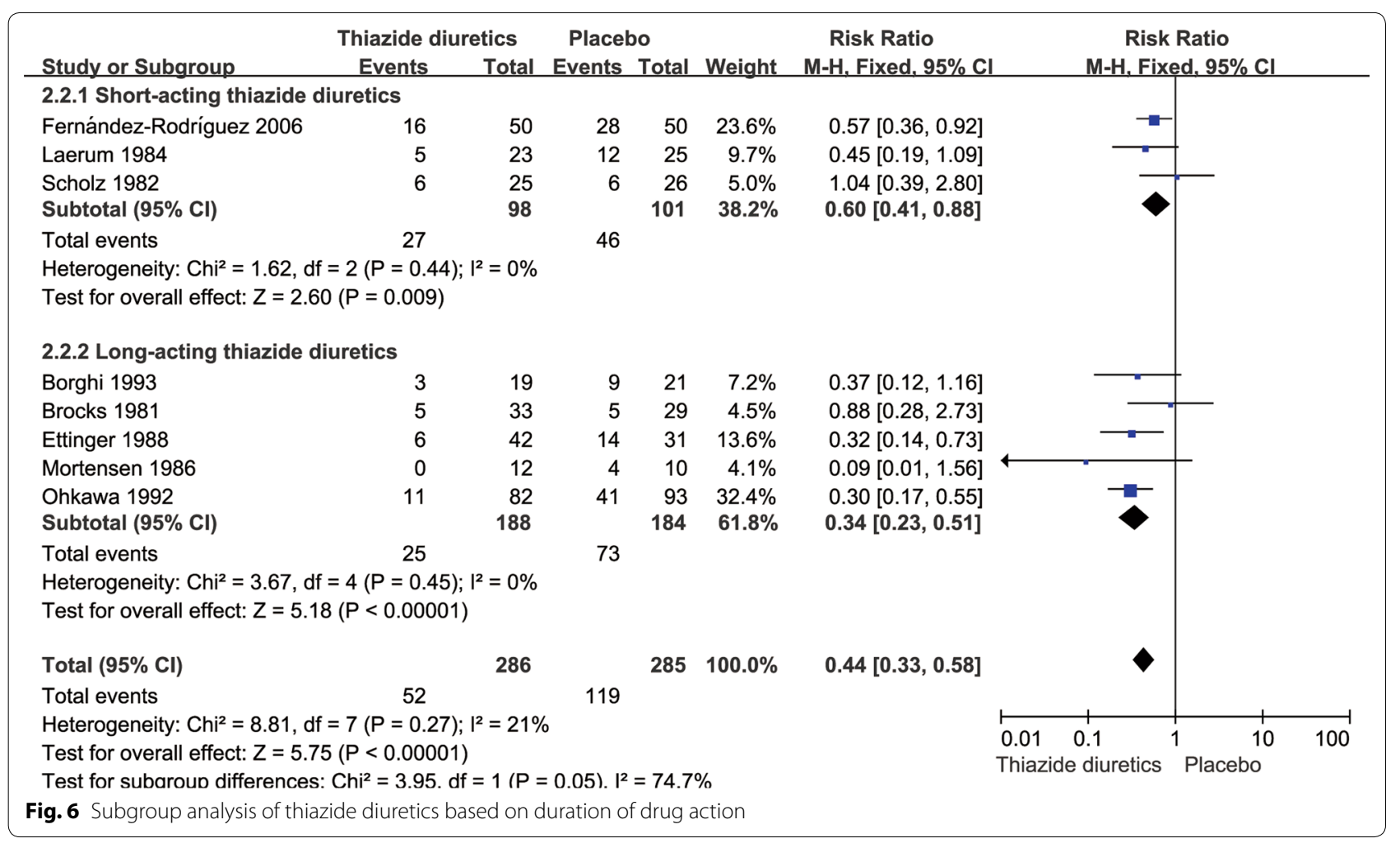




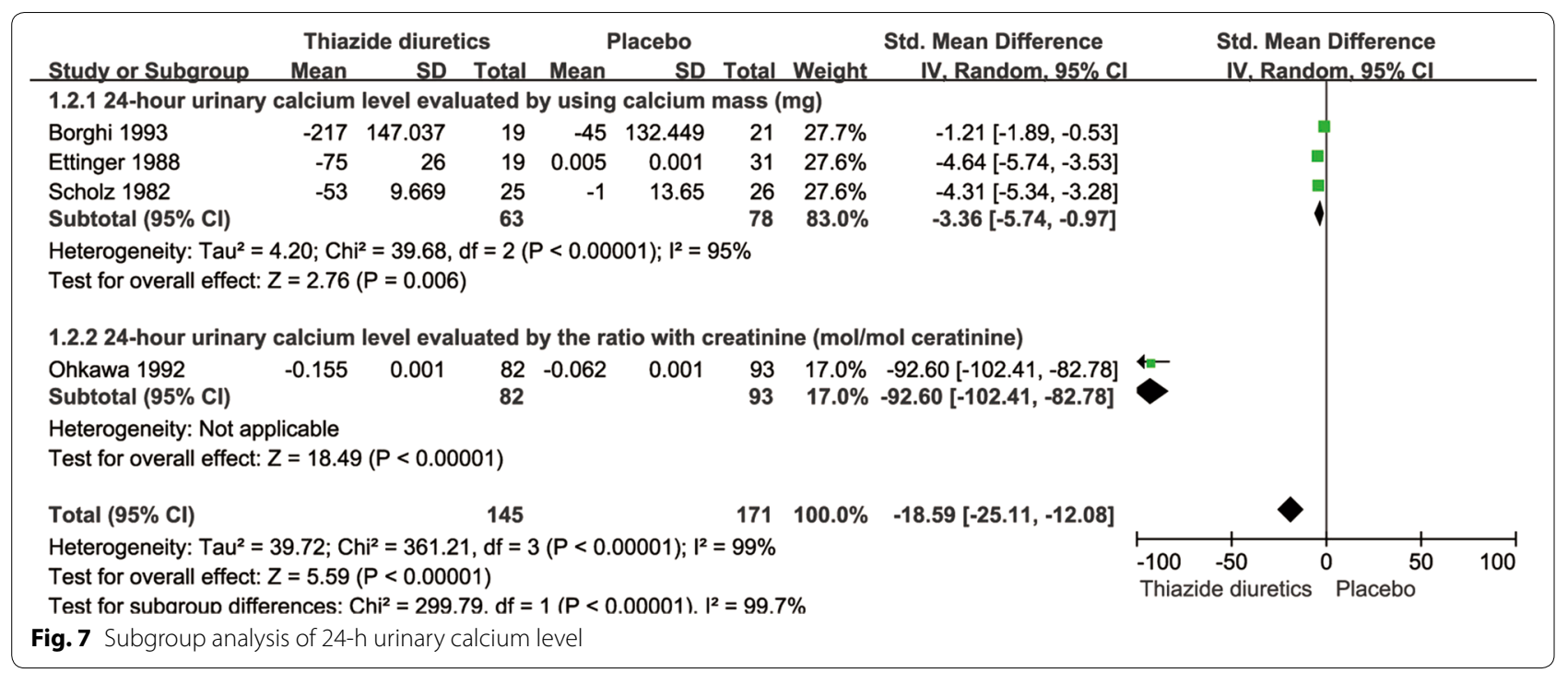

\begin{tabular}{|c|c|c|c|c|c|c|c|c|c|}
\hline & Thiazide diur & etics & Placeb & & & Risk Ratio & \multirow{2}{*}{\multicolumn{3}{|c|}{$\begin{array}{c}\text { Risk Ratio } \\
\text { M-H, Random, 95\% CI }\end{array}$}} \\
\hline Study or Subgroup & Events & Total & Events & Total & Weight & M-H, Random, $95 \%$ Cl & & & \\
\hline Borghi 1993 & 3 & 19 & 9 & 21 & $7.5 \%$ & $0.37[0.12,1.16]$ & 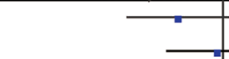 & \\
\hline Brocks 1981 & 5 & 33 & 5 & 29 & $7.7 \%$ & $0.88[0.28,2.73]$ & \multirow[b]{2}{*}{ 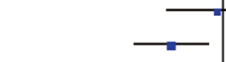 } & & \\
\hline Ettinger 1988 & 6 & 42 & 14 & 31 & $12.8 \%$ & $0.32[0.14,0.73]$ & & & \\
\hline Fernández-Rodríguez 2006 & 16 & 50 & 28 & 50 & $27.9 \%$ & $0.57[0.36,0.92]$ & $\rightarrow$ & & \\
\hline Laerum 1984 & 5 & 23 & 12 & 25 & $11.9 \%$ & $0.45[0.19,1.09]$ & \multirow[b]{3}{*}{$\varpi$} & & \\
\hline Mortensen 1986 & 0 & 12 & 4 & 10 & $1.4 \%$ & $0.09[0.01,1.56]$ & & & \\
\hline Ohkawa 1992 & 11 & 82 & 41 & 93 & $21.1 \%$ & $0.30[0.17,0.55]$ & & & \\
\hline Scholz 1982 & 6 & 25 & 6 & 26 & $9.7 \%$ & $1.04[0.39,2.80]$ & & & \\
\hline Total $(95 \% \mathrm{Cl})$ & & 286 & & 285 & $100.0 \%$ & $0.47[0.33,0.65]$ & $>$ & & \\
\hline Total events & 52 & & 119 & & & & & & \\
\hline $\begin{array}{l}\text { Heterogeneity: } \mathrm{Tau}^{2}=0.05 \\
\text { Test for overall effect: } Z=4\end{array}$ & $\begin{array}{l}h^{2}=8.81, d f=7 \\
5(P<0.00001)\end{array}$ & $7(P=0$ & $27) ;\left.\right|^{2}=2$ & $21 \%$ & & & $\begin{array}{lll}0.01 & 0.1 & 1 \\
\text { Thiazide diuretics }\end{array}$ & ${ }^{1} \frac{10}{\text { Placebo }}$ & 100 \\
\hline
\end{tabular}

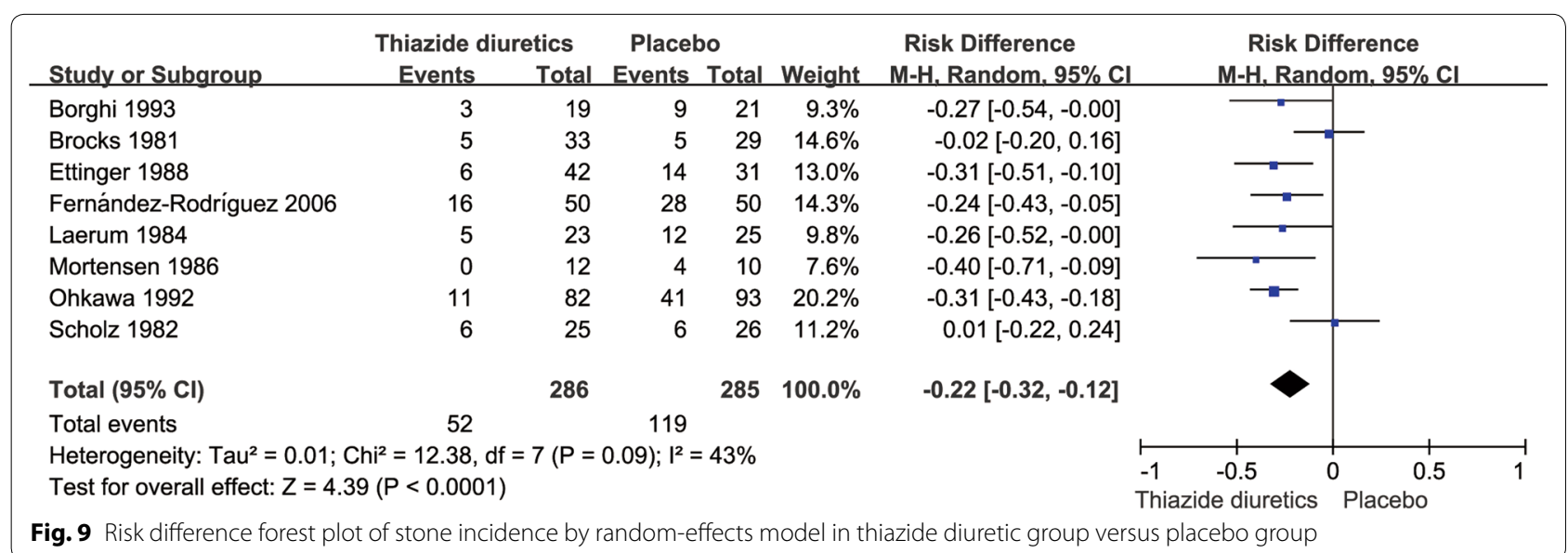




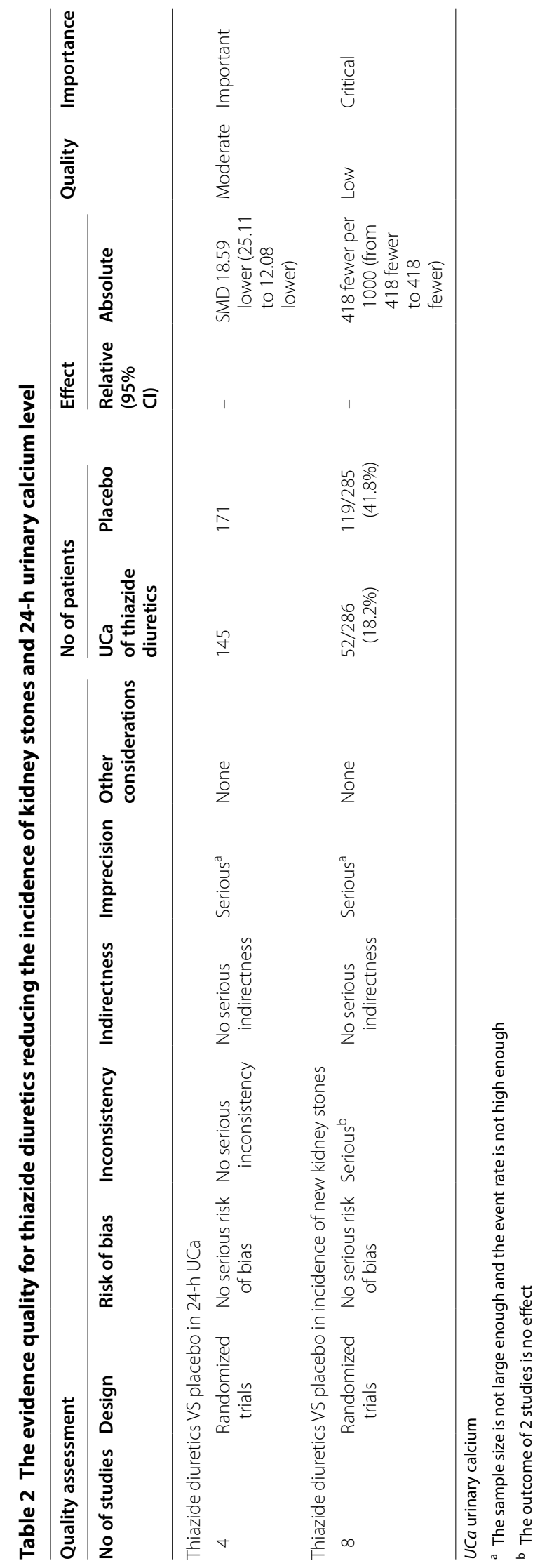


their thiazide diuretic group withdrew from the study owing to intolerance of thiazide diuretics (presence of fatigue, dizziness, and muscle symptoms); Laerum and Larsen [17] reported that two patients in their thiazide diuretic group withdrew owing to the development of hypokalemia or gout and three patients withdrew owing to the presence of mild fatigue and indigestion; Ohkawa et al. [15] mentioned that six patients in their thiazide diuretic group developed dizziness; two patients, weakness; and one patient, general malaise; and Scholz et al. reported that one patient in their thiazide diuretic group and another patient in their placebo group withdrew owing to the development of side effects. Further, 11 patients in their thiazide diuretic group complained of fatigue, nausea, and hypotension during treatment; however, the symptoms were not severe enough to cause treatment interruption [9].

\section{Publication bias}

Egger's test was performed to analyze the incidence of stone events in the patients with recurrent renal calculi treated with thiazide diuretics. The analysis revealed a $P$ value of 0.855 .

\section{GRADE rating}

The quality of evidence for thiazide diuretics in reducing the incidence of kidney calculi was low; that in reducing the 24-h urinary calcium level among the patients with recurrent renal calculi was moderate (Table 2). Further, the quality of evidence for short-acting and long-acting thiazide diuretics in reducing the incidence of kidney calculi was low (Table 3).

\section{Decision form of recommendations}

The overall strength of recommendation for the prevention of recurrent renal calculi using thiazide diuretics was not recommended (Table 4).

\section{Discussion}

Satisfactory treatment outcomes have not yet been obtained for kidney calculi, and their recurrence rate remains a concern [21-24]. Previously, multiple RCTs and reviews have reported a decreased incidence of recurrent kidney calculi with diet control (e.g., high fluid intake and calcium intake limitation) and thiazide diuretic, alkali citrate, and allopurinol administration. Herein, the overall efficacy of thiazide diuretics in reducing the incidence of recurrent renal calculi was limited when compared to the placebo and untreated groups and the pooled RD with $95 \% \mathrm{CI}$ was $-0.23(-0.30$ to -0.16 ). The quality of evidence was low owing to the insufficient samples and the inconsistent results among the studies when the GRADE criteria were used to assess the incidence of stone events. This result considerably decreased our expectation of the efficacy of thiazide diuretics in reducing the incidence of recurrent kidney calculi.

We reviewed the clinical guidelines and found that the 2016 updated edition of the Canadian Urological Association guidelines considered thiazide diuretics as a highly recommended drug for preventing recurrent kidney calculi (level of evidence: $1-3$ and grade A-B recommendation, based on the Oxford levels of evidence and grades of recommendation). However, the grade according to the American College of Physicians guidelines was weak (grade: weak recommendation, moderate-quality evidence).

In addition to concerns regarding stone events, we also observed a decrease in the serum potassium level and an increase in the uric acid level in some thiazide diuretic groups among the eight studies. Further, $3.7-20 \%$ of the patients in the thiazide diuretic groups withdrew from the trials owing to the development of adverse reactions (e.g., hypokalemia, elevated uric acid levels, and abnormal blood glucose and cholesterol levels). Clinically, the main adverse reactions of thiazide diuretics are as follows: (1) water and electrolyte disturbance, such as hypokalemia and hyponatremia; (2) cardiovascular problems, such as blood volume insufficiency and orthostatic hypotension; (3) gastrointestinal reactions, such as anorexia, nausea, gastric irritation, and constipation; (4) central nervous system problems, such as dizziness, paresthesia, and headache; and (5) abnormalities in related metabolic indicators, such as hyperglycemia and elevated total cholesterol levels [25-27]. Makam et al. [28] showed that $14.3 \%$ of thiazide users and $6.0 \%$ of non-users had adverse reactions (serum sodium level of $<135 \mathrm{mmol} / \mathrm{L}$; serum potassium level of $<3.5 \mathrm{mmol} / \mathrm{L}$; and estimated glomerular filtration rate reduction by $>25 \%$ compared with that at baseline) $(P<0.05)$. In the ALLHAT trial, the incidence of newly diagnosed diabetes was $17.1 \%$ in patients with metabolic syndrome after using chlorthalidone for 4 years; that in patients without metabolic syndrome was 7.7\% $(P<0.05)[29]$.

However, the patients' compliance was poor. Dauw et al. [30] found that when patients with kidney calculi were treated with a single prophylactic drug, the proportion of patients in the thiazide diuretic groups who followed the doctor's advice was only $42.5 \%$. Even in the presence of cardiovascular diseases (e.g., hypertension), patients in the thiazide diuretic groups were equally less docile (42\%) [31].

Taken together, thiazide diuretics yielded several adverse reactions, and the patients' compliance was low. Based on the decision table formed by the recommendation in the WHO Handbook for Guideline 


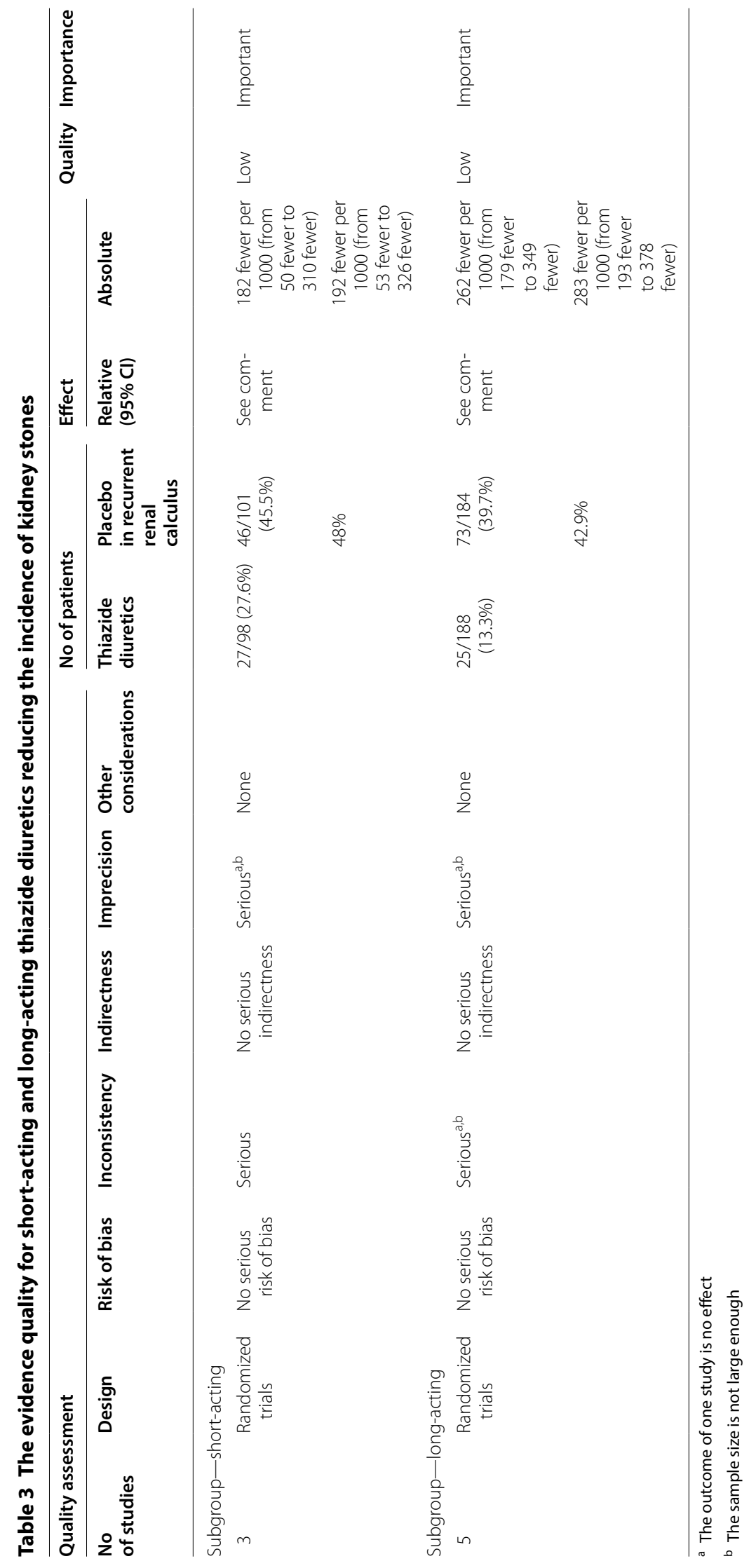


Table 4 General recommendation strength of thiazide diuretics preventing recurrent renal stones

\begin{tabular}{ll}
\hline Factor & Decision making \\
\hline $\begin{array}{l}\text { Quality of the evidence } \\
\text { (The higher the quality of the evidence, the more }\end{array}$ & Low \\
likely a strong recommendation is warranted.) & \\
Balance of benefits versus harms and burdens & Benefits and harms \\
(The larger the difference between the benefits & are balanced \\
and harms, the more likely a strong recommen- & \\
dation is warranted. The smaller the net benefit & \\
and the lower the certainty for that benefit, the & \\
more likely a conditional recommendation is & \\
warranted.) & \\
Values and preferences & \\
(The greater the variability or uncertainty in values & \\
and preferences, the more likely a conditional & \\
recommendation is warranted.) & \\
Resource use & \\
(The higher the costs of an intervention, that is, & Moderate resource- \\
the more resources consumed, the more likely a & \\
conditional recommendation is warranted.) & \\
Overall recommended strength & Low $\uparrow ?$ \\
\hline
\end{tabular}

Development and combined with the degree of benefits for patients with kidney calculi, patients' compliance, adverse reactions caused by long-term medication, and economic burden, thiazides are not recommended for use in preventing recurrent kidney calculi.

Our study also has some limitations: (1) although we searched each database using keywords, not all relevant documents were included, such as unpublished literature; (2) most of the studies included have been conducted several years ago; (3) the kidney calculi investigated in this study were all calcium stones. Whether thiazide diuretics have any effect on other types of kidney calculi remains unclear; thus, the results of this meta-analysis should be interpreted with caution; (4) some of the included studies assessed experimental groups and control groups with other measures as diet, fluid therapy or potassium chloride, which may be a source of heterogeneity; and (5) we only described the adverse reactions qualitatively and didn't conduct meta-analysis of the safety of thiazide diuretics for recurrent kidney calculi.

\section{Conclusions}

Long-term use of thiazide diuretics can reduce the incidence of recurrent renal calculi and 24-h urinary calcium level. However, the benefits are insufficient, and the quality of evidence is low. Considering the adverse effects, patients' preferences, and economic burden of long-term medication, we do not recommend the use of thiazide diuretics to prevent recurrent kidney calculi.

\section{Supplementary information}

Supplementary information accompanies this paper at https://doi. org/10.1186/s12967-020-02270-7.

Additional file 1: Table S1. Search strategy of PubMed.

Abbreviations

RCT: Randomized controlled trial; RR: Relative risk; RD: Risk difference; SMD: Standardized mean difference; $\mathrm{Cl}$ : Confidence interval.

\section{Acknowledgements}

None.

Authors' contributions

DFL, CTZ, RFZ and YLG wrote the manuscript paper. CTZ, DFL, RFZ and YLG designed the study. CTZ, DFL, YLG, HCL, RFZ and XCH collected data, performed some analysis. All authors read and approved the final manuscript.

Funding

None.

Availability of data and materials

Not applicable.

Ethics approval and consent to participate

Not applicable.

Consent for publication

Not applicable.

Competing interests

The authors declare that they have no competing interests.

\section{Author details}

${ }^{1}$ Department of Transfusion Medicine, Shanghai Jiao Tong University Affiliated Sixth People's Hospital, Shanghai 200233, China. ${ }^{2}$ Department of Laboratory Medicine, Kunshan Hospital Affiliated to Nanjing University of Traditional Chinese Medicine, Kunshan 215300, Jiangsu, China. ${ }^{3}$ School of Nursing, Shanxi Medical University, 56 Xinjian South Road, Yingze District, Taiyuan 030001, Shanxi, China.

Received: 5 August 2019 Accepted: 14 February 2020

Published online: 28 February 2020

\section{References}

1. Khan SR, Pearle MS, Robertson WG, Gambaro G, Canales BK, Doizi S, Traxer O, Tiselius HG. Kidney stones. Nat Rev Dis Primers. 2016;2:16008.

2. Qaseem A, Dallas P, Forciea MA, Starkey M, Denberg TD, Clinical Guidelines Committee of the American College of P. Dietary and pharmacologic management to prevent recurrent nephrolithiasis in adults: a clinical practice guideline from the American College of Physicians. Ann Intern Med. 2014;161:659-67.

3. Pak CY, Poindexter JR, Adams-Huet B, Pearle MS. Predictive value of kidney stone composition in the detection of metabolic abnormalities. Am J Med. 2003;115:26-32.

4. Cheungpasitporn W, Rossetti S, Friend K, Erickson SB, Lieske JC. Treatment effect, adherence, and safety of high fluid intake for the prevention of incident and recurrent kidney stones: a systematic review and metaanalysis. J Nephrol. 2016;29:211-9.

5. Goldfarb DS. Empiric therapy for kidney stones. Urolithiasis. 2019;47:107-13.

6. Nirumand MC, Hajialyani M, Rahimi R, Farzaei MH, Zingue S. Dietary plants for the prevention and management of kidney stones: preclinical and clinical evidence and molecular mechanisms. Int J Mol Sci. 2018;19:765. 
7. Dion M, Ankawi G, Chew B, Paterson R, Sultan N, Hoddinott P, Razvi H. CUA guideline on the evaluation and medical management of the kidney stone patient-2016 update. Can Urol Assoc J. 2016;10:E347-58.

8. Pearle MS, Goldfarb DS, Assimos DG, Curhan G, Denu-Ciocca CJ, Matlaga BR, Monga M, Penniston KL, Preminger GM, Turk TM, White JR. Medical management of kidney stones: AUA guideline. J Urol. 2014;192:316-24.

9. Scholz D, Schwille PO, Sigel A. Double-blind study with thiazide in recurrent calcium lithiasis. J Urol. 1982;128:903-7.

10. Brocks P, Dahl C, Wolf H, Transbol I. Do thiazides prevent recurrent idiopathic renal calcium stones? Lancet. 1981;2:124-5.

11. NICE Guideline. Renal and ureteric stones: assessment and management. BJU Int. 2019;2019(123):220-32.

12. Higgins J, Green SE. Cochrane handbook for systematic reviews of interventions, version 5.1.0 (updated March 2011). 2011. Cochrane Collaboration. http://training.cochrane.org/handbook. Accessed 22 Nov 2018.

13. Higgins JP, Thompson SG, Deeks JJ, Altman DG. Measuring inconsistency in meta-analyses. BMJ. 2003;327:557-60

14. World Health Organization. WHO handbook for guideline development https://www.who.int/publications/guidelines/handbook-for-guidelinedevelopment/zh/.

15. Ohkawa M, Tokunaga S, Nakashima T, Orito M, Hisazumi H. Thiazide treatment for calcium urolithiasis in patients with idiopathic hypercalciuria. $\mathrm{Br}$ J Urol. 1992;69:571-6.

16. Mortensen JT, Schultz A, Ostergaard AH. Thiazides in the prophylactic treatment of recurrent idiopathic kidney stones. Int Urol Nephrol. 1986;18:265-9.

17. Laerum E, Larsen S. Thiazide prophylaxis of urolithiasis. A double-blind study in general practice. Acta Med Scand. 1984;215:383-9.

18. Fernández-Rodríguez A, García-Ruiz MJ, Arrabal-Martín M, Arrabal-Polo MA, Pichardo-Pichardo S, Zuluaga-Gómez A. Papel de las tiazidas en la profilaxis de la litiasis cálcica recidivante. Actas Urológicas Españolas. 2006;30:305-9.

19. Ettinger B, Citron JT, Livermore B, Dolman LI. Chlorthalidone reduces calcium oxalate calculous recurrence but magnesium hydroxide does not. J Urol. 1988;139:679-84

20. Borghi L, Meschi T, Guerra A, Novarini A. Randomized prospective study of a nonthiazide diuretic, indapamide, in preventing calcium stone recurrences. J Cardiovasc Pharmacol. 1993;22(Suppl 6):S78-86.

21. Ferraro PM, Curhan GC, D'Addessi A, Gambaro G. Risk of recurrence of idiopathic calcium kidney stones: analysis of data from the literature. Nephrol. 2017;30:227-33.
22. Daudon M, Frochot V, Bazin D, Jungers P. Drug-induced kidney stones and crystalline nephropathy: pathophysiology, prevention and treatment. Drugs. 2018;78:163-201.

23. Wang YB, Cui YX, Song JN, Yang Q, Wang G. Efficacies of various surgical regimens in the treatment of renal calculi patients: a network metaanalysis in 25 enrolled controlled clinical trials. Kidney Blood Press Res. 2018:43:1183-98.

24. Zisman AL. Effectiveness of treatment modalities on kidney stone recurrence. Clin J Am Soc Nephrol. 2017;12:1699-708.

25. Tamargo J, Segura J, Ruilope LM. Diuretics in the treatment of hypertension. Part 1: thiazide and thiazide-like diuretics. Expert Opin Pharmacother. 2014;15:527-47.

26. Vigen R, Weideman RA, Reilly RF. Thiazides diuretics in the treatment of nephrolithiasis: are we using them in an evidence-based fashion? Int Urol Nephrol. 2011:43:813-9.

27. Zhang X, Zhao Q. Association of thiazide-type diuretics with glycemic changes in hypertensive patients: a systematic review and meta-analysis of randomized controlled clinical trials. J Clin Hypertens. 2016:18:342-51.

28. Makam AN, Boscardin WJ, Miao Y, Steinman MA. Risk of thiazideinduced metabolic adverse events in older adults. J Am Geriatr Soc. 2014;62:1039-45.

29. Black HR, Davis B, Barzilay J, Nwachuku C, Baimbridge C, Marginean H, Wright JT Jr, Basile J, Wong ND, Whelton P, et al. Metabolic and clinical outcomes in nondiabetic individuals with the metabolic syndrome assigned to chlorthalidone, amlodipine, or lisinopril as initial treatment for hypertension: a report from the Antihypertensive and Lipid-Lowering Treatment to Prevent Heart Attack Trial (ALLHAT). Diabetes Care. 2008;31:353-60.

30. Dauw CA, Yi Y, Bierlein MJ, Yan P, Alruwaily AF, Ghani KR, Wolf JS Jr, Hollenbeck BK, Hollingsworth JM. Factors associated with preventive pharmacological therapy adherence among patients with kidney stones. Urology. 2016;93:45-9.

31. Naderi SH, Bestwick JP, Wald DS. Adherence to drugs that prevent cardiovascular disease: meta-analysis on 376,162 patients. Am J Med. 2012;125(882):887.e881.

\section{Publisher's Note}

Springer Nature remains neutral with regard to jurisdictional claims in published maps and institutional affiliations.
Ready to submit your research? Choose BMC and benefit from:

- fast, convenient online submission

- thorough peer review by experienced researchers in your field

- rapid publication on acceptance

- support for research data, including large and complex data types

- gold Open Access which fosters wider collaboration and increased citations

- maximum visibility for your research: over $100 \mathrm{M}$ website views per year

At BMC, research is always in progress.

Learn more biomedcentral.com/submissions 